\title{
On the effect of short-range magnetic ordering on electron energy-loss spectra in spinels
}

\author{
D.W. McComb * \\ Department of Chemistry, University of Glasgow, Glasgow G12 8QQ \\ A.J. Craven \\ Department of Physics and Astronomy, University of Glasgow, Glasgow G12 8QQ \\ L. Chioncel and A. I. Lichtenstein \\ Institute for Theoretical Physics, University of Nijmegen, 6500 GL Nijmegen, \\ Netherlands \\ F.T. Docherty ${ }^{\dagger}$ \\ Department of Chemistry, University of Glasgow, Glasgow G12 8QQ \\ Department of Physics and Astronomy, University of Glasgow, Glasgow G12 8QQ
}

\begin{abstract}
The energy-loss near-edge structure (ELNES) at the oxygen $K$ edge in two $\mathrm{AB}_{2} \mathrm{O}_{4}$ spinels $(\mathrm{A}=\mathrm{Mg}, \mathrm{B}=\mathrm{Al}, \mathrm{Cr})$ is reported. In $\mathrm{MgAl}_{2} \mathrm{O}_{4}$ the experimental data is successfully modelled within the LDA framework. In the case of $\mathrm{MgCr}_{2} \mathrm{O}_{4}$ spin-polarisation, in the form of antiferromagnetic ordering on the Cr sub-lattice, must be included despite the fact that the measurements were performed at approximately 30 times above $T_{N}$. A model in which dynamic short-range antiferromagnetic ordering is present at room temperature is proposed to explain the results of the experiments and calculations.
\end{abstract}

Typeset using REVTEX

\footnotetext{
*Now at department of Materials, Imperial College LOndon, London SW7 2AZ. Author to whom correspondence should be addressed: dcomb@imperial.ac.uk

$\dagger$ Now at Department of Pure and Applied Chemistry, Strathclyde University, Glasgow
} 


\section{INTRODUCTION}

Electron energy-loss spectroscopy (EELS) carried out in the nano-analytical electron microscope (nano-AEM) is a powerful probe of chemistry, bonding and electronic structure in a wide range of materials. Analysis of energy-loss near-edge structure (ELNES) present on ionisation edges in EELS is now recognised as the only technique that can be used to obtain such information with close to atomic-scale spatial resolution [1]. The ELNES technique can be used to obtain information on coordination number, local symmetry and oxidation state to investigate chemical and structural inhomogeneities in advanced materials e.g. in the vicinity of interfaces, grain boundaries etc. In combination with other microscopy techniques such as electron diffraction and high resolution electron microscopy, ELNES can be utilised to obtain complete characterisation across interfaces. However the electron-specimen interactions that result in ELNES are complex. In order to advance the use of the technique it is important to conduct studies that combine fundamental investigations of the ELNES in known structures and theoretical modelling of the electron-specimen interactions.

We have previously reported the results of a combined experimental and theoretical study on a series of ternary transition metal oxides that exhibit the spinel structure [2]. The oxygen K-edge ELNES from a range of $A B_{2} O_{4}$ materials $(\mathrm{A}=\mathrm{Mg}, \mathrm{Ni}, \mathrm{Zn} ; \mathrm{B}=\mathrm{Al}$, $\mathrm{Cr}$ ) were presented and the results were analysed with the aid of the results of electronic structure calculations performed using a multiple scattering algorithm. While good agreement between experimental and theoretical spectra was obtained for $\mathrm{MgAl}_{2} \mathrm{O}_{4}$, it was extremely poor when the $\mathrm{Al}^{3+}$ cations were replaced by $\mathrm{Cr}^{3+} \cdot \mathrm{MgCr}_{2} \mathrm{O}_{4}$ undergoes antiferromagnetic ordering with a Néel temperature $\left(T_{N}\right)$ of $16 \mathrm{~K}$ [3]. An earlier study of transition metal carbides and nitrides with the rock salt structure included data from CrN, which has a Néel temperature of $288 \mathrm{~K}$. The shape of the experimental N K-edge ELNES from CrN does not change over the temperature range from $133 \mathrm{~K}$ to $433 \mathrm{~K}$ so that long-range magnetic order has no effect. Moreover, the x-ray absorption nearedge structure (XANES) matches the ELNES extremely well demonstrating that the dipole approximation holds well under the experimental conditions used to obtain the ELNES. With the exception of CrN, the agreement between experiment and theory, assuming random spins, is excellent. However, while the theoretical shape for $\mathrm{CrN}$ follows the pattern observed in the related compounds, the experimental shape from $\mathrm{CrN}$ differs significantly, indicating that some other effect is present. The agreement between experiment and theory for $\mathrm{CrN}$ is improved if the spins in the calculation are polarised to match the antiferromagnetic structure determined by Corliss et al. [5]. The reason for this is that the decrease in energy on going from the unpolarised to the polarised state is $336 \mathrm{meV}$ per formula unit so that the thermal energy available at $433 \mathrm{~K}$ is unlikely to destroy the polarisation over the short range even though the long range order disappears. This observation led us to explore the effect of the antiferromagnetic coupling between the chromium sites on the calculated ELNES to see if the agreement with experiment was improved despite the fact that the measurements were performed well above the Néel temperature. 


\section{MATERIALS AND METHODS}

The spinel specimens were synthesised from the parent binary oxides using standard solid-state procedures. The lattice parameters, oxygen parameter, $u$, and degree of inversion, $\lambda$, were obtained by Rietveld refinement of X-ray or neutron diffraction data. The $\mathrm{MgCr}_{2} \mathrm{O}_{4}$ specimen was identified as cubic spinel, $a=8.33202(1) \AA$, with an oxygen parameter, $u$, of 0.26124 which is close to the ideal value of 0.25 . Rietveld refinement of the site occupancies revealed the sample to be $100 \%$ normal $(\lambda=0) . M g \mathrm{Al}_{2} \mathrm{O}_{4}$ was also identified as cubic spinel, $a=8.08098(2) \AA$, with an oxygen parameter close to the ideal value $(u=0.26116)$. Rietveld refinement of the site occupancies revealed the sample to be partially inverse $(\lambda=0.32)$. Samples were prepared for EELS by crushing the powder and then dispersing the particles in propan-2-ol. A few drops of the suspension were then placed on holey carbon film coated grid and the solvent was allowed to evaporate. The EELS data were collected using a VG HB5 cold field emission gun (FEG) scanning transmission electron microscope (STEM), operated at $100 \mathrm{kV}$. The spectra were recorded using a Gatan 666 parallel electron energy-loss spectrometer. A collection half-angle of $12 \mathrm{mrad}$ was used, defined by a $1 \mathrm{~mm}$ aperture preceding the STEM detectors, and a spray aperture was positioned after the same detectors to remove any stray scattering from the collector aperture. Spectra were recorded at an energy-dispersion of $0.1 \mathrm{eV} /$ channel, using a $1 \mathrm{~nm}$ electron probe containing $0.2 \mathrm{nA}$ with a convergence half-angle of $11 \mathrm{mrad}$.

The ab-initio electronic structure calculations were carried out using the scalar relativistic linear muffin-tin orbital (LMTO) method within the atomic-sphere approximation in two flavours: LDA and $\mathrm{LDA}+\mathrm{U}[6,7]$. The MT-radii used in all calculations were $2.32,2.44$ and 1.95 a.u. for $\mathrm{Mg}, \mathrm{Cr}$ and $\mathrm{O}$, respectively. Two additional classes of empty spheres were introduced with $R=1.82$ a.u. and $R=1.66$ a.u. respectively. The frozen core approximation was employed in the generation of the potential and the parametrization of von Barth-Hedin [8] was used for the exchange correlation part of the effective one-electron potential, obtained within the local spin density approximation of the density functional theory. Convergence of charge density was achieved within $10^{-5}$. The integration over the Brillouin zone has been done by the tetrahedron method with $64 \mathrm{k}$-points for the Self-consistent field calculation. Although all of the Cr atoms in the normal spinel structure are crystallographically equivalent, in the spin-polarised calculation they were treated differently because of the antiferromagnetic coupling. In the spinel structure, the Cr ions form a corner shared tetrahedral lattice, comprising the $16 d$ sites in space group $F d 3 m$, that is normally referred to as the pyrochlore lattice. In the calculations, an antiferromagnetic structure in which two of $\mathrm{Cr}$ atoms among four $\mathrm{Cr}$ atoms in each tetrahedron of the pyrochlore lattice are in one orientation of magnetization while the other two $\mathrm{Cr}$ atoms are aligned in the opposite orientation. Although the actual magnetic structure of $\mathrm{MgCr}_{2} \mathrm{O}_{4}$ is likely to be more complex than this simplified model, this antiferromagnetic structure was taken as the initial state of the calculation and was preserved after the self-consistent calculation. 


\section{RESULTS AND DISCUSSION}

The intensity observed in an electron energy-loss spectrum is related to the product of an atomic transition matrix element and the symmetry-projected unoccupied DOS on the atomic site of interest. Over the limited energy range of interest for analysis of fine structure on an ionisation edge, typically $<15 \mathrm{eV}$, it is reasonable to assume that the transition matrix element is a slowly varying function. Thus, to a first approximation, it is reasonable to compare the observed ELNES with the broadened siteand symmetry-projected unoccupied DOS obtained from the theoretical calculations [1]. The calculated DOS is convoluted with two functions; a Lorentzian function whose width varies with energy above the threshold to simulate the broadening from the finite lifetime of the excited state and a Gaussian $(F W H M=0.8 \mathrm{eV})$ to account for the experimental resolution at the energy-loss of the oxygen K-edge. The lifetime broadening is approximated by two contributions. One is from the lifetime of the core hole, which can be obtained from atomic data $(0.11 \mathrm{eV}$ for oxygen $1 \mathrm{~s})[9]$, and the other is from the lifetime of the excited atomic electron. The latter can be estimated using the expression of Muller et al assuming a valence band width of $18 \mathrm{eV}$ and a plasmon energy of $25 \mathrm{eV}$ $[10]$.

In Figure 1 the experimental oxygen K-ELNES of $\mathrm{MgAl}_{2} \mathrm{O}_{4}$ is compared with the result of the LDA calculation. In this calculation the oxygen K-edge was simulated assuming that the spinel structure was $100 \%$ normal. Although experimental data was recorded from a partially inverse material, it was concluded in our earlier study that the effect of inversion on the oxygen K-edge was minimal and hence this was a valid assumption [2]. This conclusion is supported by the excellent agreement between experiment and theory demonstrated in Figure 1. The result of the LDA calculation is consistent with the calculated band structure results reported by other workers using different algorithms.

When the octahedral site cation is changed from $\mathrm{Al}^{3+}$ to $\mathrm{Cr}^{3+}$ the agreement between experiment and theory is much poorer (Figure 1). The results of the neutron diffraction studies reported previously clearly demonstrate that the $\mathrm{MgCr}_{2} \mathrm{O}_{4}$ specimen is $100 \%$ normal [2]. This is as expected since placing the $\mathrm{Cr}^{3+}$ ion in a tetrahedral environment would be energetically unfavourable due to the large Jahn-Teller distortion that would result from unsymmetric occupation of the frontier orbitals [12]. This is significant since it establishes that the discrepancy between experiment and theory is not associated with structural inversion.

In the initial LDA calculations (Fig. 1), it was assumed that the band structure of the material is independent of electron spin. However, it is well established that this is not necessarily true in a transition metal system where spin-ordering on the metal sites could influence the band structure through the exchange-interaction term. It is also possible that the low dispersion of the d-band in the vicinity of the Fermi level may induce strong electron correlation effects and thus invalidate the independent electron model. The former hypothesis could be investigated by introducing spin polarisation into the calculation while the latter requires incorporation of electron correlation effects via the $\mathrm{LDA}+\mathrm{U}$ approach.

The calculated symmetry-projected density of states (DOS) on the anion and cation 
sites are compared in Figure 2. The upper panel shows the oxygen $p$ - and the chromium $d$-DOS obtained from the LDA calculation performed with no spin polarisation. In this non-magnetic structure it is clear that there is a large DOS at the Fermi level $\left(E_{F}\right)$ and there is no distinct energy gap $\left(E_{g}\right)$. This is a classic situation where the total energy of this "metallic" structure could be lowered by spin polarisation. In the LSDA calculation, a structure that exhibits antiferromagnetic ordering of the electron spins on the chromium sites has been assumed and the $\mathrm{O} p$ - and $\mathrm{Cr} d-$ DOS obtained are shown in the middle panel of Figure 2. Analysis of the $\mathrm{Cr} d-$ DOS shows that the energy of one of the spin states $(\uparrow)$ has shifted to lower energy while the other $(\downarrow)$ has shifted to higher energy. This has resulted in the creation of an energy gap of $0.7 \mathrm{eV}$ between the valence and conduction bands. The moments on the two $\mathrm{Cr}$ sites were found to be $2.69 \mu_{B}$ and $-2.69 \mu_{B}$ in this antiferromagnetic insulator and as expected the total energy was found to be significantly less, $0.811 \mathrm{eV} / \mathrm{Cr}$, than the non-magnetic structure. It should be noted that if the $\mathrm{Cr}$ cations are antiferromagnetically ordered then two magnetically inequivalent oxygen sites must also be present in the structure. In a normal spinel such as $\mathrm{MgCr}_{2} \mathrm{O}_{4}$, each $\mathrm{O}$ site has four nearest neighbours $(1 \mathrm{Mg}$ \& $3 \mathrm{Cr}$ ) in a tetrahedral arrangement. Consequently in the magnetic structure $50 \%$ of the oxygens will have two $\mathrm{Cr}$ neighbours with parallel and one with antiparallel spin alignments and the other half of the oxygens will have the opposite arrangement. This means the $p$ - DOS on each oxygen site is slightly different as shown in Figure 2. When the AF ordering on the chromium sub-lattice is reversed the $p$ - DOS on each type of oxygen site will also change. However, the symmetry of the lattice and perfect AF ordering means that the effect here is simply a switching of the $p$ - DOS between the two types of oxygen site.

In the "LSDA+U" a value of $\mathrm{U}=2 \mathrm{eV}$ has been used and the same antiferromagnetic ordering of the Cr sites, as used in the LSDA calculation, has been assumed. The general shape and interpretation of the site and symmetry projected DOS predicted by the $\mathrm{LSDA}+\mathrm{U}$ calculation is very similar to that obtained from the LSDA calculation. (Fig. 2) The main difference is that the magnitude of the energy gap has now increased to $1.3 \mathrm{eV}$, an effect that is expected since the main role of the Hubbard $\mathrm{U}$ is to stabilise the magnetic structure. In the "LSDA+U" results, the magnetic moments on the Cr sites are similar to those found in the LSDA calculation and lattice energy was $1.476 \mathrm{eV} / \mathrm{Cr}$ less than the non-magnetic lattice energy obtained from a $\mathrm{LDA}+\mathrm{U}$ calculation. Once again the larger stabilisation energy in the LSDA $+U$ calculation can be attributed directly to the effect of $U$. Nevertheless it is clear that the antiferromagnetic state is more favourable than non-magnetic structure for the experimental lattice parameters used in the calculation.

Since there are two types of oxygen site in the magnetic structures it was necessary to calculate the average density of states by summing the $p$ - DOS for each spin state. Both types of oxygen site have the same average $p$ - DOS. The $p$ - DOS obtained from each theoretical model was broadened as described earlier and compared with the experimental ELNES data. (Fig. 3) It is immediately apparent that changing from the non-magnetic structure in the LDA calculation to the antiferromagnetically ordered structure in the LSDA calculation results in a dramatic improvement in the agreement between theory and experiment. Further refinement of the calculation to 
include electron correlation effects has only a minor (detrimental) effect on the observed shape.

The implication of these results is that the oxygen K-ELNES (and hence the electronic structure) of $\mathrm{MgCr}_{2} \mathrm{O}_{4}$ can be successfully modelled as a magnetically ordered insulator. It is known that $\mathrm{MgCr}_{2} \mathrm{O}_{4}$ exhibits antiferromagnetic behaviour below its Néel temperature of $16 \mathrm{~K}$. However, the ELNES measurements were conducted at room temperature where all long-range antiferromagnetic order has been lost. The only possible explanation of these observations is that even in the room temperature paramagnetic state there exists short-range (on the order of 1-2 unit cell lengths) antiferromagnetic ordering of the spin directions on the chromium sites over a period of time longer than the interaction time between this region and the fast electron. Since the electron velocity is $\simeq 10^{8} \mathrm{~m} \mathrm{sec}^{-1}$ and the dimensions of the region are $\simeq 1 \mathrm{~nm}$, the interaction time is $\simeq 10^{-17} \mathrm{sec}$.

Thus, in order to explain the experimental and theoretical results obtained we are proposing that (a) short range $B-B$ site antiferromagnetic correlations exist in $\mathrm{MgCr}_{2} \mathrm{O}_{4}$ at room temperature, and (b) these correlations can be detected by electron energy-loss spectroscopy. Let us consider each of these hypotheses in more detail. The fact that $\mathrm{MgCr}_{2} \mathrm{O}_{4}$ shows antiferromagnetic behaviour at all demonstrates that the crystal can reduce its lattice energy by ordering the electron spins on the $B$-sites. In the absence of any radical change in crystal structure or lattice parameter it follows that correlation of the electron spins will lower the lattice energy of the solid at every temperature below the melting point. However, at temperatures above $T_{N}$, the thermal energy will act to constantly randomise the long-range order and average out any correlations. However, given a difference in energy of $811 \mathrm{meV} / \mathrm{Cr}$, it would be expected that spin correlations would persist at temperatures as high as $9 \times 10^{3} \mathrm{~K}$. Consequently the important factor is the length of time over which the correlations exist - this will depend on temperature, decreasing as the temperature increases. The distance over which the correlations exist will also depend on temperature - for a fixed time interval the spatial extent of the ordering will decrease as the temperature increases.

To our knowledge, the existence of short-range order (SRO) in $\mathrm{MgCr}_{2} \mathrm{O}_{4}$ has not been investigated in detail previously. However, SRO in zinc ferrite, $Z n F e_{2} \mathrm{O}_{4}$, has been investigated by several research groups. The comparison between $\mathrm{ZnFe} \mathrm{O}_{4}$ and $\mathrm{MgCr}_{2} \mathrm{O}_{4}$ is an excellent one since both materials can be synthesised as $100 \%$ normal cubic spinels. $\mathrm{ZnFe}_{2} \mathrm{O}_{4}$ is also an antiferromagnetic insulator, with high spin $\mathrm{Fe}\left(3 d^{5}\right)$, and a low ordering temperature $\left(T_{N}=10.5 \mathrm{~K}\right)[13]$. The spin structure of the material as a function of temperature is complex with dynamic SRO reported to persist up at least $10 T_{N}$. This conclusion was reached on the basis of neutron scattering [14,15], Mossbauer resonance [14,16], and muon-spin-rotation/relaxation ( $\mu-\mathrm{SR}$ ) (Ref. [14]) studies. Below $T_{N}$, long range order (LRO) has been observed in $\mathrm{ZnFe}_{2} \mathrm{O}_{4}$ by a number of research groups. However, at temperatures up to $\simeq 10 T_{N}$, broad maxima have been observed and a model in which small antiferromagnetic regions exhibit "superantiferromagnetic" behaviour due to SRO has been proposed [15]. It has been deduced from the width of the maxima that the size of the domains exhibiting SRO is of the order of 3nm [14]. The results of the $\mu-\mathrm{SR}$ studies clearly demonstrate that the SRO is dynamic rather than static, a conclusion supported by recent single crystal neutron scattering studies 
[17], with an estimated minimum fluctuation rate of $1.5 \mathrm{GHz}$, although the error on this estimate could be as large as the value itself [14]. This rate corresponds to a correlation time $\left(\tau_{c}\right)$ of less than $6.6 \times 10^{-10} \mathrm{~s}$. Since the SRO is detectable by neutron scattering, it is also possible to derive an upper value for the correlation time from the interaction time between a neutron and a domain of this size. Assuming an energy range 0.1 $1 \mathrm{eV}$ we obtain neutron velocities in the range $0.45-1.4 \times 10^{4} \mathrm{~ms}^{-1}$. Using the estimated domain size from previous studies of $3 \mathrm{~nm}$, we conclude that in order for SRO to be detected by neutron scattering the correlation time must be greater than $3 \times 10^{-13} \mathrm{~s}$. This is four orders of magnitude greater than the interaction time for $10^{5} \mathrm{eV}$ electrons and so it can be concluded that electron energy-loss spectroscopy is potentially sensitive to much shorter lived correlations.

The final question that must be considered is the length scale over which the correlation must extend in order for the domain to appear "infinite" as far as the electron (or $\mathrm{X}$-ray) excitation process is concerned. This is readily deduced from the multiple scattering theory developed to describe the X-ray absorption near-edge structure (XANES), which is the X-ray equivalent of ELNES. This real space multiple scattering approach gives the same result as a band structure calculation in reciprocal space provided that both calculations converge [18]. The real space multiple scattering approach to calculation of electronic structure has been shown to be robust and reliable provided a large enough cluster is used to ensure convergence. Based on our own work on spinels, we have demonstrated that convergence in $\mathrm{MgAl}_{2} \mathrm{O}_{4}$ is achieved, at the energy resolution available in ELNES or XANES, with a cluster radius of less than $0.7 \mathrm{~nm}$ [2]. Thus the correlation of the spin has only to exist over a distance of $\simeq 2 \mathrm{~nm}$ for a time $\simeq 10^{-17} \mathrm{sec}$ to have a large effect on the edge shape in ELNES or XANES.

In summary, we have demonstrated that the oxygen K-edge ELNES of $\mathrm{MgCr}_{2} \mathrm{O}_{4}$ cannot be simulated using electronic structure calculations performed within the LDA. However, reasonable agreement between experiment and theory is achieved when the calculation is performed within the LSDA framework and full antiferromagnetic ordering on the $\mathrm{Cr}$ sites is included in the model. While the magnetic structure of $\mathrm{MgCr}_{2} \mathrm{O}_{4}$ is likely to be more complex than the model assumed, the improved agreement between theory and experiment obtained using this model shows that spin polarisation plays a key role in defining the electronic structure as determined by EELS. While studies of the magnetic behaviour of $\mathrm{MgCr}_{2} \mathrm{O}_{4}$ above $\mathrm{T}_{\mathrm{N}}$ have not been reported in the literature, the work performed by other groups on $\mathrm{ZnFe}_{2} \mathrm{O}_{4}$, also an antiferromagnetic (AF) insulator, clearly demonstrate the presence of short range AF ordering well above $T_{N}$. We conclude that electron energy-loss spectroscopy is a highly sensitive probe of such ordering and can potentially reveal the presence of AF order over distances of $\simeq 2 \mathrm{~nm}$. 


\section{REFERENCES}

[1] R.F. Egerton, Electron energy-loss spectroscopy in the electron microscope, 2nd edition (Plenum, New York, 1996)

[2] F.T. Docherty, A.J. Craven, D.W. McComb, and J.Skakle, Ultramicroscopy 86, $273(2001)$

[3] H. Shaked, J.M. Hastings, and L.M. Corliss, Phys. Rev. B 1, 3116 (1970).

[4] A.T. Paxton, M. van Schilfgaarde, M. MacKenzie, and A.J. Craven, J. Phys.: Condens. Matter 12, 729 (2000).

[5] L.M. Corliss, N. Elliot and J.M. Hastings Phys. Rev. 117, 929 (1960)

[6] O.K. Andersen, Phys. Rev. B 12, 3060 (1975); O.K. Andersen and O Jepsen, Phys. Rev. Lett. 53, 2571 (1984); O.K. Anderson, O. Jepsen and D. Glotzel in, Highlights of Condensed Matter Physics, edited by F. Bassini, F. Fumi and M.P. Tosi (NorthHolland, New York, 1995); R.O. Jones and O. Gunnarson, Rev. Mod. Phys. 61, 689 (1989).

[7] V.I. Anisimov, J. Zannen and O.K. Andersen, Phys. Rev. B 44, 943 (1991); V.I. Anisimov, I.V. Solovyev, M.A. Korotin, M.T. Czyzyk and G.A. Sawatzky, Phys. Rev. B 48, 16929 (1993); A.I. Lichtenstein, J. Zannen and V.I. Anisimov, Phys. Rev. B 52, R5467 (1995); V.I. Anisimov, F. Aryasetiawan, A.I. Lichtenstein, J. Phys.: Condens. Matter 9, 767 (1997).

[8] U. von Barth and L Hedin, J. Phys. C 5, 1629 (1972).

[9] J. Stohr, NEXAFS Spectroscopy (Springer, New York, 1996)

[10] D.A. Muller, D.J. Singh, and J. Silcox, Phys. Rev. B 57, 8181 (1998).

[11] S. Kostlmeier, and C. Elsasser, Phys. Rev. B 60, 14025 (1999); K. van Benthem and H. Kohl, Micron 31, 347 (2000).

[12] P.A. Cox, Transition Metal Oxides: An Introduction to their Electronic Structure and Properties (Oxford University Press, Oxford, 1995).

[13] U. Konig, E.F. Bertaut, Y. Gros, M. Mitrikov, and G. Chol, Solid State Commun. 8,759 (1970).

[14] W. Schiessl, W. Potzel, H. Karzel, M. Steiner, G.M. Kalvius, A. Martin, M.K. Krause, I. Halevy, J. Gal, W. Schafer, G. Will, M. Hillberg, and R. Wappling, Phys. Rev. B 53, 9143 (1996).

[15] Yu.G. Chukalkin and V.R. Shtirts, Sov. Phys. Solid State 30, 1683 (1988).

[16] S.A. Oliver, H.H. Hamdeh, and J.C. Ho, Phys. Rev. B 60, 3400 (1999).

[17] K. Kamazawa, Y. Tsunoda, K. Odaka, and K. Kohn, J. Phys. Chem. Solids 60, 1261 (1999).

[18] A.L. Ankudinov, B. Ravel, J.J. Rehr, and S.D. Conradson, Phys. Rev. B 58, 7565 (1998). 


\section{FIGURES}

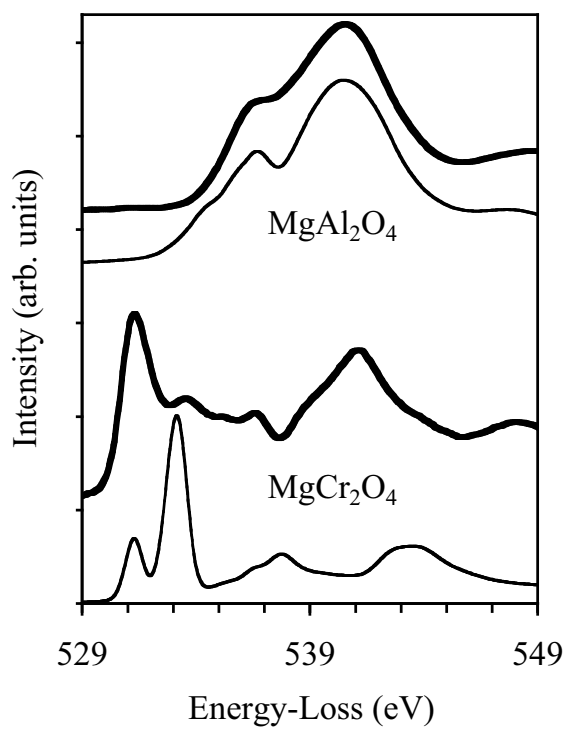

FIG. 1. Comparison of experimental oxygen K-ELNES data (thick lines) with the broadened p-projected unoccupied DOS on the oxygen site obtained from LDA calculations (thin lines) for $\mathrm{MgAl}_{2} \mathrm{O}_{4}$ and $\mathrm{MgCr}_{2} \mathrm{O}_{4}$. In each case the first peak in the theoretical result has been aligned to the first peak in the experimental spectrum.

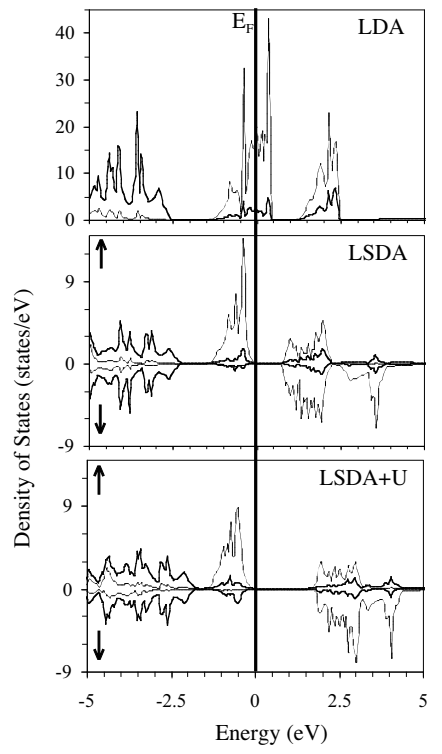

FIG. 2. Comparison of the calculated oxygen p-DOS (thick line) and chromium d-DOS (thin line) in $\mathrm{MgCr}_{2} \mathrm{O}_{4}$ obtained from different theoretical models. The Fermi energy is at $0 \mathrm{eV}$. The upper panel shows the result for the non-magnetic structure. In the LSDA (middle panel) and LSDA+U (lower panel) calculations, antiferromagnetic ordering on the Cr sites has been assumed and the result for each spin state on one of the $\mathrm{Cr}$ sites is shown. The density of states on the second $\mathrm{Cr}$ site is inversely related to the first due to the antiferromagnetic ordering. 


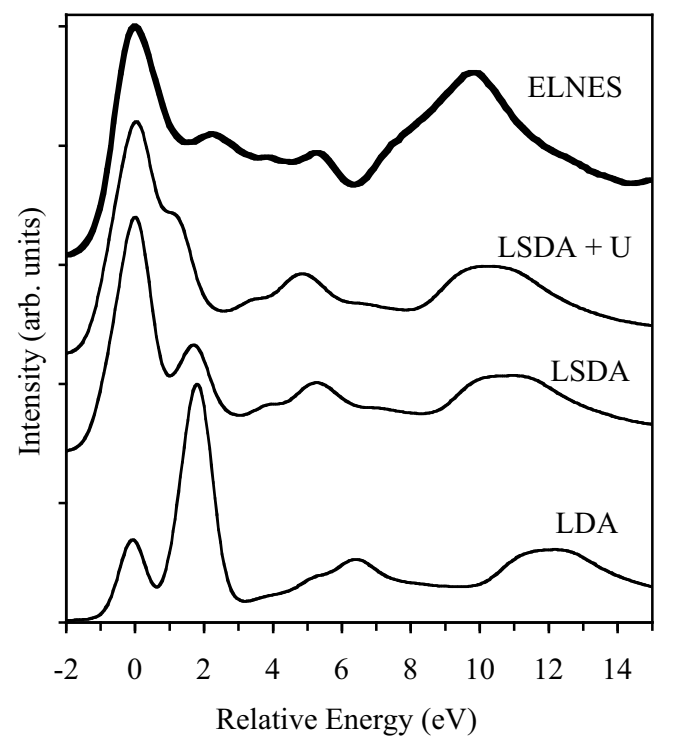

FIG. 3. Comparison of the broadened oxygen p-DOS with the experimental oxygen K-ELNES data for $\mathrm{MgCr}_{2} \mathrm{O}_{4}$. The first peak in each spectrum has been aligned to $0 \mathrm{eV}$. Where antiferromagnetic ordering is present the spectrum is derived from the average of the "spin-up" and "spin-down" DOS. 